ISSN: 2316-6517

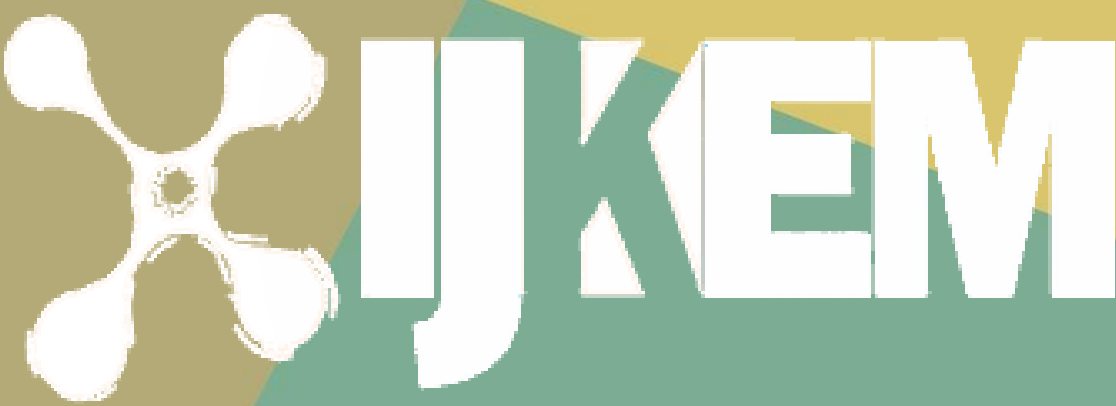

International Journal of Knowledge Engineering and Management

v. 9, n. $25,2020$.

(c) (1) (8)

ijkem.ufsc.br 
Florianópolis, v. 09, n. 25, p. 127 - 143, 2020.

- ISSN 2316-6517 •

- DOI: 1047916 •

\title{
COMPARTILHAMENTO DE CONHECIMENTO EM UMA PEQUENA EMPRESA DE PRODUTOS ORTOPÉDICOS: ANÁLISE DAS PERCEPÇÕES DOS COLABORADORES
}

\author{
FERNANDO SOARES DA ROCHA JÚNIOR \\ Doutorando em Engenharia e Gestão do Conhecimento - EGC \\ Universidade Federal de Santa Catarina (UFSC) \\ rochavix@gmail.com \\ ORCID: https://orcid.org/0000-0002-7236-5303 \\ VÂNIA MENEGHINI DA ROCHA \\ Mestrado em Educação - Univille \\ Instituto Federal Catarinense (IFC) \\ vania.rocha@ifc.edu.br \\ ORCID: https://orcid.org/0000-0002-7468-2994 \\ MARCELO MACEDO \\ Doutorado em Engenharia e Gestão do Conhecimento - EGC \\ Universidade Federal de Santa Catarina (UFSC) \\ marcelo5369@gmail.com \\ ORCID: https://orcid.org/0000-0003-4154-9318
}

Submissão: 26/05/21. Aceitação: 12/07/21. Sistema de avaliação: duplo cego (double blind review).

UNIVERSIDADE FEDERAL DE SANTA CATARINA (UFSC)

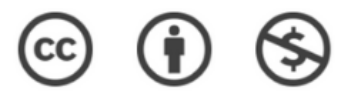


Florianópolis, v. 09, n. 25, p. 127 - 143, 2020.

- ISSN 2316-6517 •

- DOI: $1047916 \cdot$

\section{COMPARTILHAMENTO DE CONHECIMENTO EM UMA PEQUENA EMPRESA DE PRODUTOS ORTOPÉDICOS: ANÁLISE DAS PERCEPÇÕES DOS COLABORADORES}

\section{Resumo}

Objetivo: O objetivo central deste artigo é identificar as principais práticas de compartilhamento de conhecimento por meio da visão dos colaboradores de uma pequena empresa de produtos ortopédicos, considerando suas vivências e percepções diárias nas atividades da organização.

Design|Metodologia|Abordagem: A pesquisa é desenvolvida a partir de uma abordagem qualitativa, utilizando-se de entrevista semiestruturada. Para a análise de resultados, foi utilizada a técnica de análise temática de Braun e Clarke (2012).

Resultados: Os principais resultados encontrados demonstram que 0 compartilhamento de conhecimento na empresa foco do estudo é realizado por intermédio da utilização das tecnologias de comunicações comuns ao público, assim como, com a realização frequente da técnica de brainstorming, conversas rápidas e outras estratégias para resolução de problemas conforme identificados na pesquisa.

Originalidade|Valor: O presente estudo destaca-se devido à escassez de estudos práticos relacionados a gestão do conhecimento em empresas de pequeno porte, caracterizando assim, uma lacuna de pesquisa para investigações empíricas.

Palavra-chave: Compartilhamento de Conhecimento; Pequena Empresa; Tecnologias; Brainstorming. 
Florianópolis, v. 09, n. 25, p. 127 - 143, 2020.

- ISSN 2316-6517 •

- DOI: $1047916 \cdot$

\section{KNOWLEDGE SHARING IN A SMALL ORTHOPEDIC PRODUCTS COMPANY: ANALYSIS OF EMPLOYEES' PERCEPTIONS}

\section{Abstract}

Goal: The main objective of this article is to identify the main knowledge sharing practices through the vision of employees of the small company of orthopedic products, considering their daily experiences and perceptions of the organization's activities.

Design/Methodology/Approach: The research is developed from a qualitative approach, using a semi-structured interview. For the analysis of results, the thematic analysis technique of Braun and Clarke (2012) was used.

Results: The main results found demonstrate that the sharing of knowledge in the company that is the focus of the study is carried out through the use of communication technologies common to the public, as well as, with the frequent performance of the brainstorming technique, quick conversations and other problem-solving strategies as identified in the survey.

Originality/Value: This study stands out due to the scarcity of practical studies related to knowledge management in small businesses, thus characterizing a research gap for empirical investigations.

Keywords: Knowledge Sharing; Small business; Technologies; Brainstorming. 
Florianópolis, v. 09, n. 25, p. 127 - 143, 2020.

- ISSN 2316-6517 •

- DOI: 1047916 .

\section{Introdução}

O presente estudo busca contribuir com a construção e expansão dos conhecimentos empíricos relacionados as práticas de compartilhamento de conhecimento em pequenas organizações. Dito isso, foi desenvolvido um estudo empírico em uma empresa de produtos ortopédicos de Joinville, o qual, será mais detalhado na sequência.

Nesta perspectiva, a pesquisa realizada, envolve a interligação de 3 constructos importantes para seu desenvolvimento. Para isso, serão abordados neste trabalho, aspectos voltados a gestão do conhecimento, compartilhamento de conhecimento, e por fim, serão estudadas as percepções dos colaboradores de uma pequena empresa de ortopedia de Joinville/SC, buscando compreender como ocorre o compartilhamento de conhecimento na organização, como também, analisar os principais efeitos ou impactos que o compartilhamento dos saberes gera na rotina da empresa.

Para compreender as percepções dos colaboradores em relação as suas maneiras de compartilhar o saber, foi elaborada a seguinte questão de pesquisa, a qual norteará esse estudo em sua plenitude: De que maneira ocorre o compartilhamento de conhecimento na empresa Alfa ${ }^{1}$ na percepção de seus colaboradores?

De modo geral, este trabalho está organizado em seções, como segue: A primeira seção destina-se à introdução. Em seguida, na segunda seção, apresenta-se a revisão teórica de literatura atualizada sobre o tema, trazendo aporte teórico de diversos autores, tais como: Alavi e Leidn (2001); Na Ubon e Kimble (2002); Drucker (1993); Dalkir (2005); Gold, Malhotra e Segars (2001); Takeuchi e Nonaka (2008); Cummings (2003); Santos e Rados (2020); Gray (2006); Ngah e Ibrahim (2010); Utami, Rofik, Cahyaningtyas, Darminto (2021); Allameh, Khozani e Baniasadi (2020); e Harel, Schwartz e Kaufmann (2020). A terceira seção é dedicada a prévia descrição sobre a metodologia utilizada para a elaboração do trabalho. Na sequência, a quarta seção abrange os principais resultados encontrados e suas respectivas análises, e, ao final, são realizadas as considerações finais relacionadas aos principais achados da pesquisa, constituindo assim, a quinta e última seção do artigo.

\footnotetext{
${ }^{1}$ nome fictício atribuído à empresa foco do estudo
} 


\section{Gestão do Conhecimento}

Para Alavi e Leidn (2001), a gestão do conhecimento existe para aproveitar ao máximo o conhecimento em uma organização. Preocupa-se em identificar e potencializar o conhecimento coletivo objetivando fornecer as organizações maiores vantagens competitivas.

De acordo com Na Ubon e Kimble (2002, p. 02), a Gestão do Conhecimento, refere-se a gestão dos processos que envolvem "criação, disseminação e utilização do conhecimento por meio da fusão de tecnologias, estruturas organizacionais e pessoas para criar o aprendizado, a resolução de problemas e a melhores decisões em uma organização". Partindo dessa definição, torna-se possível verificar as principais atividades ligadas a gestão do conhecimento organizacional, as quais, estão intrinsecamente relacionadas, ao gerenciamento dos ativos intangíveis das organizações.

Na concepção de Drucker (1993), o conhecimento pode ser percebido como um recurso estratégico, pelo qual, muitas organizações, obtém através deste, mais sustentabilidade e maior poder de competitividade no mercado. Para as organizações que realmente valorizam os conhecimentos adquiridos, inúmeros desafios se colocam para todos os tipos e níveis empresariais, dentre os quais, estão atrelados ao gerenciamento efetivo desses ativos intangíveis que são os conhecimentos que a maioria das organizações possuem, contudo, a maior parte delas, não desenvolveram competência para gerenciá-los adequadamente. Neste sentido, a GC $^{2}$ procura oferecer métodos ou processos para alcance de uma maior vantagem competitiva, ou simplesmente auxiliar, na utilização correta e mais eficaz desses recursos estratégicos fundamentalmente importantes no mundo moderno.

Partindo desse pressuposto, a gestão do conhecimento, que se materializa por meio da implementação eficaz de um sistema de gestão do conhecimento, de fato, deve ser pensada, planejada e operacionalizada observando os vários desafios observados internamente em empresas públicas ou privadas, como também, atender de maneira

\footnotetext{
${ }^{2}$ gestão do conhecimento
} 
eficiente, os detalhes, características ou necessidades específicas de cada organização.

Para Dalkir (2005, p. 06), a GC "é uma mistura surpreendente de estratégias, ferramentas e técnicas, algumas das quais, não são nenhuma novidade" nas práticas educacionais e empresariais. Conforme explanado, a GC se utiliza de técnicas mercadológicas já existentes para uma melhor organização no contexto do conhecimento, dentro das organizações.

Gold, Malhotra e Segars (2001, p. 186), corroboram que, os alicerces inerentes ao conhecimento, tais como, "tecnologia, estrutura e cultura, juntamente com a aquisição, conversão, aplicação e proteção de conhecimento, são capacidades organizacionais essenciais para um melhor desempenho organizacional". Um Sistema de Gestão do Conhecimento deve sempre atentar para se estruturar sob três aspectos centrais, estão entre ele: a) pessoas; b) processos; e, c) tecnologias.

De acordo com Takeuchi e Nonaka (2008, p. 19), a GC pode ser compreendida com um "processo de criar continuamente novos conhecimentos, disseminando-os amplamente através da organização e incorporando-os velozmente em novos produtos/serviços, tecnologias e sistemas".

A ISO 30401:2018, de 2018, tem uma definição parecida de GC, a qual sinaliza que se trata uma abordagem composta pela criação, compartilhamento e aplicação de conhecimento com foco na ampliação de produtividade, do rendimento e do avanço organizacional. Desta maneira, entende-se que o compartilhamento de conhecimento é um dos processos estruturais e basilares de um eficiente sistema de gestão do conhecimento.

Para uma implementação coerente e mais adaptada as necessidades organizacionais de um $\mathrm{SGC}^{3}$ nas organizações, torna-se necessário, em especial, um planejamento minucioso e adequado a realidade organizacional, respeitando assim, as intrínsecas variáveis identificadas em cada organização de maneira singular. Nesse sentido, o follow-up ou acompanhamento de perto dessas atividades são importantes para mensurar a eficácia de um SGC. Para Kayas e Wright (2018), um sistema de gestão do conhecimento, é percebido pela gestão como uma forma de alavancar

\footnotetext{
${ }^{3}$ sistema de gestão do conhecimento
} 
informações para melhorar o desempenho através da identificação dos incontáveis ativos intangíveis e gerenciá-los mais eficientemente.

\subsection{Compartilhamento de Conhecimento}

Para ocorrer com qualidade um processo de compartilhamento de conhecimento, não se deve pensar apenas como se o compartilhar fosse um processo unicamente comunicativo, entretanto, devem ser observados outros fatores intrínsecos e não menos importantes, os quais, de alguma maneira, influenciam diretamente ou indiretamente os resultados da troca de conhecimentos envolvendo alguns processos de aprendizagens (Cummings, 2003).

Na concepção de Santos e Rados (2020, p. 21), estamos entrando na era da sociedade 5.0, na qual, as coisas, tecnologias, conhecimentos, entre outras realidades, deverão ser compartilhados para a obtenção do bem comum: trata-se de "uma sociedade de conhecimento com uma visão de futuro focada no compartilhamento, de modo a proporcionar benefícios e prosperidade para o gênero humano".

De acordo com Cummings (2003), alguns fatores podem impactar significativamente na implementação do sistema de compartilhamento do conhecimento nas organizações. Segundo o autor, os fatores impactantes, dentre os quais, são essenciais para o processo de compartilhamento de conhecimento no contexto organizacional são: a) confiança entre as pessoas; b) devem existir locais específicos para fomentar o processo - $\mathrm{Ba}^{4}$; c) os receptores do conhecimento devem desejar aprender; d) compartilhar de maneira correta os novos conhecimentos adquiridos e, por último; e) a organização deve providenciar um ambiente propício para que as trocas de conhecimento aconteçam de um jeito eficiente e eficaz. Os fatores impactantes defendidos por Cummings (2003) são imprescindíveis para o efetivo compartilhamento de conhecimento, entre os já citados, destaca-se, de maneira especial, o desenvolvimento de um ambiente propício que estimule e encoraje um compartilhamento de saberes sem receios de penalidades e perseguições das chefias.

$\mathrm{Na}$ visão de Kayas e Wright (2018), o processo de compartilhamento de conhecimento, depende intrinsecamente, de pré-condições culturais, que são a princípio

\footnotetext{
${ }^{4}$ locais específicos para trocas de conhecimentos e experiências
} 
adequadas em esferas intraorganizacionais, pelas quais, se forem corretamente direcionadas, podem viabilizar assim, as benéficas trocas de conhecimentos.

Em se tratando de pequenas e médias empresas, a partilha do conhecimento é um pilar importante na GC, pois, oferece as devidas atualizações das tarefas da rotina organizacional, como também, auxilia no desenvolvimento dos processos organizacionais emergentes.

Nesse sentido, o subprocesso de GC, denominado de compartilhamento de conhecimento, é geralmente, bastante utilizado em organizações micro ou pequenas, por intermédio de uma comunicação aberta e contínua, de forma tácita, muitas vezes sem os colaboradores se darem conta disso, contudo, sendo utilizada com notável frequência por todos os colaboradores (Gray, 2006).

No que diz respeito aos aspectos salutares, juntamente com a percepção dos autores Ngah e Ibrahim (2010), os efeitos do compartilhamento na performance das pequenas e médias organizações são positivos, contudo, a quantidade restrita de pesquisas a respeito deixa um bom espaço para novas discussões e intervenções. Os efeitos do compartilhamento de conhecimento também auxiliam na inovação e desenvolvimento gradativo de pequenos negócios (Utami et al. 2021). Na visão de Allameh, Khozani e Baniasadi (2020, p. 48), "o compartilhamento de conhecimento tem um efeito direto sobre as capacidades dinâmicas das pequenas organizações".

Em um recente estudo com entrevistas semiestruturadas (face-to-face), realizado com 202 pequenas empresas em Israel, os pesquisadores, Harel, Schwartz e Kaufmann (2020), descobriram que se as pequenas empresas industriais desejarem obter uma maior ganho mercadológico em competitividade e inovação, deveriam implantar, gradativamente, processos de compartilhamento de conhecimento em suas respectivas organizações. $\mathrm{Na}$ análise e visão dos autores, eles enfatizam que o compartilhamento de conhecimento melhora o potencial de inovação, ajuda no desenvolvimento e melhoramento de produtos, promove um melhor nível de desempenho no marketing e aumenta a performance organizacional como um todo.

\section{Procedimentos metodológicos}


No que concerne aos procedimentos metodológicos, este estudo utiliza como pilar principal a abordagem qualitativa de pesquisa que, atualmente, é reconhecida pela sua relevância na pesquisa acadêmica. Trata-se de um procedimento de pesquisa que agrega diferentes concepções filosóficas importantes para investigação dos diferentes fenômenos (Creswell, 2007).

Em relação ao paradigma de pesquisa escolhido para esse estudo, a visão de vida escolhida foi interpretativista (Morgan, 1980), a qual, pressupõe, uma análise minuciosa da opinião ou da percepção de determinado fenômeno por parte dos participantes envolvidos na investigação e, que enxerga, conforme Merriam (2009), a realidade de maneira subjetiva com o ponto de vista individual de cada ser humano. Em relação a tipologia utilizada na pesquisa, esse aspecto será clarificado na sequência.

No que se refere a tipologia de estudo, foi escolhida a pesquisa qualitativa básica, a qual, de acordo Merriam (2009), tem como característica principal a identificação e interpretação dos significados que são importantes para os indivíduos.

A técnica escolhida para a para a coleta de dados da presente pesquisa qualitativa básica é denominada entrevista semiestruturada, a qual, terá como essência, o propósito de atender as expectativas relacionadas ao objetivo principal do estudo com perguntas abertas, dentre as quais, serão posteriormente analisadas e compreendidas conforme as percepções dos entrevistados em relação ao compartilhamento de conhecimento que é desenvolvido dentro da organização pesquisada.

Como elemento estrutural e direcionador da entrevista semiestruturada, foi criado e aplicado pelo autor, um roteiro de entrevista, pelo qual foram direcionadas as conversas com 05 colaboradores participantes da pesquisa.

Por fim, como método de análise de dados, para esse estudo, será utilizado a análise temática de Braun e Clarke (2012). Em resumo, a Figura 01, demonstra o passo a passo da metodologia utilizada para o desenvolvimento do presente trabalho. 
Florianópolis, v. 09, n. 25, p. 127 - 143, 2020.

- ISSN 2316-6517 •

- DOI: 1047916 •

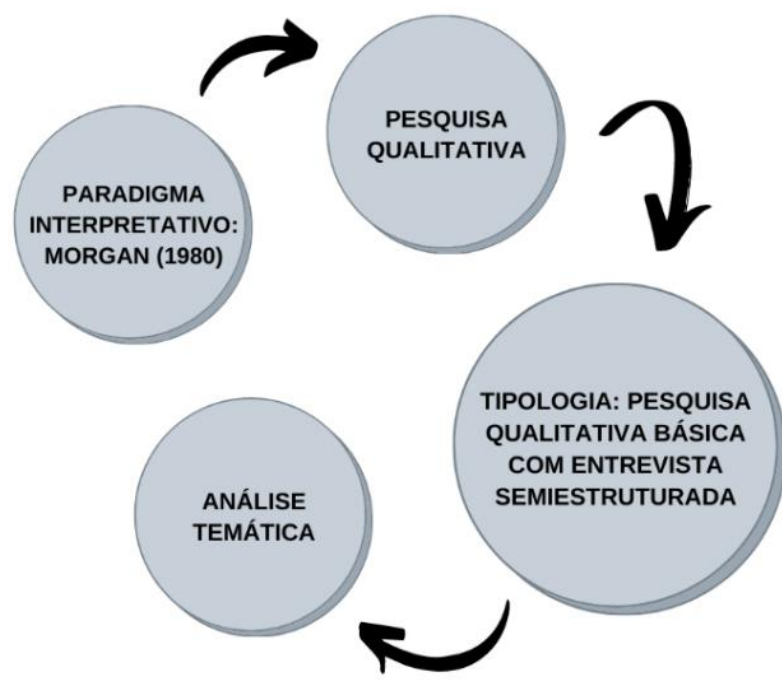

Figura 01 - Metodologia utilizada para estruturação da pesquisa

Fonte: elaborado pelos autores (2021)

\section{Resultados e discussões}

As percepções dos colaboradores verificadas neste trabalho foram sistematizadas e correlacionadas às categorias para uma melhor visualização. Sendo assim, as informações detalhadas estão dispostas no quadro 1, o qual, evidencia, de maneira sucinta, as principais características e descobertas do estudo.

Quadro 1 - Categorias e características encontradas na análise dos dados

\begin{tabular}{|c|l|}
\hline Categorias & \multicolumn{1}{|c|}{ Características } \\
\hline $\begin{array}{c}\text { Perspectiva } \\
\text { Bidirecional }\end{array}$ & $\begin{array}{l}\text { Foi identificado por meio da percepção dos colaboradores da empresa } \\
\text { Alfa que o compartilhamento de conhecimento é tido como um processo } \\
\text { bidirecional. }\end{array}$ \\
\hline $\begin{array}{c}\text { Compartilhamento } \\
\text { contínuo }\end{array}$ & $\begin{array}{l}\text { É uma prática comum na organização o compartilhamento de } \\
\text { conhecimento contínuo. }\end{array}$ \\
\hline $\begin{array}{c}\text { "Ba" do } \\
\text { conhecimento }\end{array}$ & $\begin{array}{l}\text { Na organização analisada, existe um espaço comum para as pessoas } \\
\text { trocarem conhecimentos específicos da empresa. }\end{array}$ \\
\hline Brainstorming & $\begin{array}{l}\text { A técnica de brainstorming é utilizada entre a equipe e de maneira } \\
\text { continua para incentivar a resolução de problemas e também para } \\
\text { discutir as estratégias de aumento das vendas dos produtos. }\end{array}$ \\
\hline Apoio da Gestão & $\begin{array}{l}\text { Os colaboradores têm total liberdade para compartilhamento das } \\
\text { informações relacionadas aos processos organizacionais. }\end{array}$ \\
\hline Percepção do & $\begin{array}{l}\text { Todos os colaboradores, incluindo os sócios-gestores da empresa, } \\
\text { entendem, que o conhecimento é fator fundamental para o aumento de } \\
\text { impacto do }\end{array}$ \\
\hline
\end{tabular}




\begin{tabular}{|c|l|}
\hline $\begin{array}{c}\text { conhecimento para } \\
\text { organização }\end{array}$ & $\begin{array}{c}\text { Para a maioria dos colaboradores, os processos de compartilhamento } \\
\text { de conhecimento são eficientes. No entanto, um(a) colaborador(a) com } \\
\text { maior experiência na área, indicou a necessidade da implantação de } \\
\text { um software ERP para melhoramento no de } \\
\text { prompartilhamento } \\
\text { administrativos da organização. }\end{array}$ \\
\hline $\begin{array}{c}\text { Artefatos } \\
\text { Os colaboradores enfatizaram o uso frequente das mídias sociais para } \\
\text { compartilhamento de conhecimento e marketing/propaganda da } \\
\text { empresa. }\end{array}$ \\
\hline $\begin{array}{c}\text { Transferência de } \\
\text { Conhecimento }\end{array}$ & $\begin{array}{l}\text { Em relação ao aspecto relacionado a transferência de conhecimento } \\
\text { por meio das tecnologias/mídias sociais, os entrevistados(as) alegaram } \\
\text { que as tecnologias disponíveis atualmente, são fundamentais para } \\
\text { compartilhar, vender e promover os produtos/serviços oferecidos ao } \\
\text { mercado. }\end{array}$ \\
\hline $\begin{array}{c}\text { Excesso de } \\
\text { informações e } \\
\text { conhecimentos diz respeito ao excesso de informações e conhecimentos } \\
\text { acessíveis com as tecnologias, a minoria dos colaboradores enfatizou } \\
\text { que é um desafio gerenciar os excessos enfrentados por meio do uso } \\
\text { das tecnologias disponibilizadas. }\end{array}$ \\
\hline
\end{tabular}

Fonte: elaborado pelos autores (2021)

Em relação a perspectiva de troca de conhecimento verificada na empresa, foi verificada, por meio das respostas dos colaboradores, que se trata de uma organização fundamentalmente democrática, na qual, todos os colaboradores (as), sem exceção, manifestaram ter liberdade total para expor suas percepções, fragilidades, pensamentos, dicas, implementar melhorias, trocar conhecimentos e fazer o que for necessário para o aumento da eficácia organizacional. No que se refere ao compartilhamento contínuo de conhecimento, a gestão da organização promove e incentiva frequentemente que os colaboradores compartilhem, conversem e troquem informações importantes relacionadas as vendas, serviços prestados, novos produtos, clientes, etc. É perceptível que os sócios-gestores criam diariamente um ambiente com "espírito livre", saudável e propício, que auxilia positivamente nas ações de compartilhamento de conhecimento.

Em se tratando de espaço para compartilhamento de conhecimento, existe uma sala, na qual, são realizados encontros rotineiros para troca de informações importantes e onde são compartilhados também, para todos os colaboradores, os conhecimentos e atividades diárias da organização. Esse espaço pode ser considerado um " $B a$ " do conhecimento pois possui as características inerentes, conforme teorias existentes sobre os referidos espaços. A técnica de brainstorming, é utilizada com todos os colaboradores e ajuda na resolução de problemas e na criação de novos processos 
elencados à prestação de serviços com mais qualidade, e sobretudo, auxilia para o efetivo envolvimento e participação de todos os colaboradores, nas decisões e rumos importantes para a empresa.

Em relação ao suporte gerencial oferecido aos colaboradores, foi possível identificar com a presente análise, que existe uma profunda ligação e apoio gerencial por parte dos sócios da empresa, o que possibilita, uma maior confiabilidade por parte dos colaboradores na gestão da empresa, o que impacta, em uma maior sustentabilidade na troca de informações importantes relacionadas aos processos organizacionais. Foi percebido também, que o ambiente organizacional, de acordo com os entrevistados(as), é harmônico e saudável tanto para os funcionários(as), quanto para os sócios-gestores da empresa. Acerca da percepção do impacto do conhecimento para a organização, todos os colaboradores foram unânimes em relação a este ponto específico, enfatizando que conhecimento e troca de conhecimentos são primordiais para a manutenção da empresa no mercado, assim como, tem efeito salutar em sua competitividade e no seu core business da empresa.

Ao que se refere ao processo de compartilhamento na empresa e suas percepções de melhorias, a maioria dos colaboradores estão satisfeitos de como é realizado. Entretanto, uma colaboradora enfatizou a importância da implantação de um Enterprise Resource Planning (ERP), sistema integrado de gestão, na qual, em sua percepção, agilizaria os processos gerais da administração da empresa, como também, auxiliaria na gestão "de informações para uma coleta de dados mais completa de nossos clientes". Na opinião dessa colaboradora em especial, com um sistema $E R P$, a empresa teria um gerenciamento mais eficaz dos dados e informações relacionados aos clientes, e, impactaria, na produção de conhecimentos importantes para melhor atender o mercado. Para divulgação, vendas e marketing da empresa, os artefatos tecnológicos utilizados, dentre os quais, foram informados pelos colaboradores, estão: Instagram, Skype, Facebook, Whatsapp e E-mail. As mídias sociais ajudam na prospecção, contato, vendas e no acompanhamento em tempo real das necessidades dos clientes. Na percepção dos colaboradores, as mídias sociais representam um papel fulcral no desempenho da organização e no compartilhamento instantâneo de novos conhecimentos relacionados aos produtos, serviços, clientes e futuros clientes.

Tendo em consideração a transferência de conhecimento entre pessoas e tecnologias, mais uma vez, as mídias sociais, são, na percepção dos funcionários (as), 
fundamentais para as vendas dos produtos, follow-up dos clientes, contato rápido com fornecedores, networking, e contribui significativamente, para uma gestão mais eficaz de toda a cadeia produtiva. Quanto ao excesso de informações, esse aspecto se caracterizou com um ponto crítico, sendo citado por uma colaboradora que impacta negativamente em alguns processos da organização, ao salientar que "as pessoas têm acesso a muitas informações, entretanto, em muitos momentos, não possuem tempo para pensar nas possíveis soluções". Por se tratar de uma pequena empresa, esse aspecto ainda não impacta na gestão e no compartilhamento de conhecimento entre gestão e seu o staff (ainda não afeta na troca de conhecimento em membros da equipe).

\section{Conclusões}

Com este trabalho, foi possível reafirmar a importância do compartilhamento de conhecimento em uma pequena organização de produtos ortopédicos. Foram verificados, por meio da pesquisa, aspectos teóricos e práticos importantes relacionados à gestão do conhecimento, mais especificamente no processo de compartilhamento de conhecimento.

No que diz respeito aos aspectos teóricos, neste caso específico, estão relacionados a escassez de artigos ou trabalhos que focalizam o compartilhamento de conhecimento em pequenas organizações, neste sentido, ressalta-se a importância teórica e contributiva deste estudo para o encorajamento de novas análises focadas em pequenas empresas.

Em relação aos aspectos práticos, esta análise contribuiu com informações e conhecimentos atualizados da realidade do compartilhamento de conhecimento em uma organização privada, ao demonstrar de maneira clara, de acordo com a percepção dos seus colaboradores, as suas posições relacionadas ao tema, suas práticas de compartilhamento e valorização do conhecimento, e por fim, a necessidade cada vez maior, de ter processos de gestão do conhecimento dentro das organizações, sejam estas, pequenas, médias ou grandes empresas.

Para concluir, torna-se necessário enfatizar a escassez de estudos práticos relacionados à gestão do conhecimento, de maneira geral, em pequenas empresas, caracterizando um gap de pesquisa para investigações empíricas futuras. 
Florianópolis, v. 09, n. 25, p. 127 - 143, 2020.

-ISSN 2316-6517 •

- DOI: $1047916 \cdot$

\section{Referências}

Alavi, M., \& Leidn, D. E. (2001). Knowledge management and knowledge management systems: Conceptual foundations and research issues. MIS Quarterly, 25, 107-136.

Allameh, S. M.; Khozani, M. K.; Baniasadi, B. (2020) Consequences of knowledge processes in small businesses: the role of knowledge acquisition, dynamic capabilities, knowledge sharing and creativity. Int. J. Process Management and Benchmarking, Vol. 10, No. 1.

Braun, V.; Clarke, V. (2012) Thematic analysis. In: Cooper, H. et al. (Eds.). APA handbook of research methods in psychology, v. 2, Research designs: Quantitative, qualitative, neuropsychological, and biological. Washington, DC: American Psychological Association, v. 2 p. 57-71.

Creswell, J. W. (2007) Projeto de pesquisa: Métodos qualitativo, quantitativo e misto. 2. ed. Porto Alegre: Artmed.

Cummings, J. (2003) Knowledge Sharing: A Review of the Literature. Washington D. C.: The World Bank Operations Evaluation Department.

Dalkir, K. (2005) Knowledge Management in Theory and Practice. Oxford, UK: Elsevier.

Drucker, P. F. (1993) Sociedade Pós-Capitalista. São Paulo: Pioneira.

Gold H. A.; Malhotra, A; Segars, A, H.; (2001). Knowledge management: An Organizational Capabilities Perspective. Journal of Management Information Systems, $18(1), 185-214$.

Gray, C. (2006). Absorptive capacity, knowledge management and innovation in entrepreneurial small firms. International Journal of Entrepreneurial Behavior \& Research, 12 (6),345-360.

Harel, R.; Schwartz, D.; Kaufmann, D. (2020) Sharing knowledge processes for promoting innovation in small businesses. European Journal of Innovation Management.

ISO 30401:2018. (2018) International Organization For Standardization. Knowledge Management Systems - Requirements. Genebra: ISO.

Kayas, O, G.; Wright, G. (2018) Knowledge Management and Organisational Culture. The Palgrave Handbook of Knowledge Management. ISBN 978-3-319-71434-9 (eBook). 
Florianópolis, v. 09, n. 25, p. 127 - 143, 2020.

- ISSN 2316-6517 •

- DOI: 1047916 •

Merriam, S. B. (2009) Qualitative research: a guide to design and implementation. San Francisco: Jossey-Bass/Wiley, 2009.

Morgan, G. (1980) Paradigms, metaphors, and puzzle solving in organization theory. Administrative Science Quarterly, v. 25, n. 4, p. 605-622.

Na Ubon, A.; Kimble, C. (2002) Knowledge Management in Online Distance Education. Proceedings of the 3rd International Conference Networked Learning, University of Sheffield, UK, pp. 465-473.

Ngah e Ibrahim (2010). The Effect of Knowledge Sharing on Organizational Performance in Small and Medium Enterprises.

Santos, N.; Rados, G. J. V. (2020) Fundamentos teóricos de gestão do conhecimento. E-book. - 1. ed. - Florianópolis: Pandion, 114 p.

Takeuchi, H.; Nonaka, I. (2008) Criação e dialética do conhecimento. In: Takeuchi, H.; Nonaka, I. Gestão do conhecimento. Porto Alegre: Bookman.

Utami, Y.; Rofik, M.; Cahyaningtyas, N. W.; Darminto, D. P. (2021). Impact of Knowledge Sharing and Innovation on Small Business Performance. CISIS 2020: Complex, Intelligent and Software Intensive Systems pp 408-411. 